\title{
Ultrasonic radiation from wedges of cubic profile: Experimental results
}

Brian E. Anderson,,${ }^{\mathrm{a},{ }^{*}}$ Marcel C. Remillieux, ${ }^{\mathrm{a}}$ Pierre-Yves Le Bas, ${ }^{\mathrm{a}}$ and T. J. Ulrich, ${ }^{\mathrm{a}}$ and Lukasz Pieczonka, ${ }^{\mathrm{b}}$

${ }^{a}$ Geophysics Group (EES-17), MS D446, Los Alamos National Laboratory, Los Alamos, NM 87545, USA

${ }^{b}$ Department of Robotics and Mechatronics, AGH University of Science and Technology, Al. A. Mickiewicza 30, 30-059 Krakow, Poland

${ }^{*}$ Corresponding author, E-mail address: bea@lanl.gov (B. E. Anderson)

\begin{abstract}
This paper presents experimental results demonstrating the increase in ultrasonic radiation obtained from a wedge of cubic profile relative to a plate of uniform thickness. The wedge of cubic profile provides high efficiency sound radiation matching layer from a mounted piezoelectric transducer into the surrounding air. Previous research on structures with indentations of power-law profile has focused on vibration mitigation using the so called "acoustic black-hole" effect, whereas here such structures are used to enhance ultrasonic radiation. The work provides experimental verification of the numerical results of Remillieux et al. [Ultrasonics 54(5), 1409 (2014)].
\end{abstract}

Keywords: Piezoelectric Transducers; Air-Coupled Ultrasound; Acoustic-Structure Interaction; Time-Reversal Acoustics. 


\section{Introduction}

Efficient radiation of ultrasonic sound, for the purpose of noncontact nondestructive evaluation and testing applications, presents unique challenges [1]. Piezoelectric transducers, which can be easily designed to have resonance frequencies in the ultrasonic frequency range $(>20 \mathrm{kHz})$, suffer from a large impedance contrast on the order of $10^{5}$ for radiation into air. Nonetheless, piezoelectric transducers are relatively inexpensive and have the capacity to generate a large amount of energy. Coupling the vibration energy produced by a piezoelectric transducer into acoustic radiation into air can be made more efficient with the use of matching layers [1]. Here we present results from an unconventional matching layer.

The "acoustic black-hole" effect obtained from structures with indentations of power law profiles has been used for vibration mitigation, with expected applications in the aerospace and automotive industries [2]-[3]. This effect was first evidenced by Mironov [4] and Krylov [5], who showed theoretically that a flexural wave slows down and eventually stops when traveling down a wedge with a thickness decreasing smoothly to zero according to a power law. As a result, the flexural wave is not reflected from the wedge tip and the elastic energy becomes trapped in the thinnest portion of the wedge. In practice, however, manufacturing a perfect wedge with a tip of zero thickness is not feasible, which is detrimental to the absorptive properties of the wedge. In addition, the length of the wedge should be comparable to or larger than the acoustic wavelength targeted, which restricts its use above $2 \mathrm{kHz}$ in industrial applications [3]. 
Rather than using plates of power-law profile for vibration mitigation, Remillieux et al. recently proposed the idea of using these structures as a matching layer for coupling more efficiently the energy emitted by a piezoelectric transducer into air [6]. They used 2D numerical simulations to compare the radiation from wedges of quadratic, cubic, and quartic profiles. They found that the cubic and quartic profiles provided more efficient sound radiation than a quadratic profile, but that the quartic profile wedge did not provide a significant increase in efficiency over the cubic profile to warrant machining the more difficult quartic profile. The cubic profile increased the sound radiation by a factor of $5.5(14.8 \mathrm{~dB})$ over the sound radiated by the piezoelectric transducer alone. It is noted that this comparison was made at the respective peak resonance frequencies for each configuration, but the cubic profile introduced several strong resonances, whereas the bare piezoelectric radiation only possessed one resonance frequency.

The purpose of this paper is to provide the first experimental verification of the idea of using wedges of power-law profiles to enhance ultrasonic radiation of piezoelectric transducers into air. Wedges of linear, quadratic, and cubic profiles were manufactured and piezoelectric transducers were mounted to them. Experimental measurements of their sound radiation are compared to radiation from a piezoelectric transducer mounted to a thin plate and to a transducer mounted to a thick plate. Each of the wedge assemblies provide more efficient ultrasonic radiation compared to the thin and thick plates. Further, the experiments show that the wedge of cubic profile provided more efficient radiation than those of linear and quadratic profiles as was also demonstrated in the work of Remillieux et al [6], despite the fact that the model was restricted to two dimensions, whereas the wedges used here have a finite width in the third dimension, giving rise to the possibility of structural modes in this third dimension. 
The goal of this work is to develop a highly efficient radiator of sound spanning the frequency range of $20 \mathrm{kHz}$ to $100 \mathrm{kHz}$. One of the intended applications for these radiators is to use them as the primary sources in a Time Reversal Acoustic Non-Contact Excitation (TRANCE) source [7]. TRANCE relies on the focusing properties of the time reversal process [8]-[9] and is used to excite structures without contact for nondestructive evaluation purposes. In terms of excitation amplitude, a preliminary and unoptimized design of TRANCE has been shown to be more efficient by nearly an order of magnitude than traditional sources, such as a focused ultrasound transducer. Figure 1 displays a photo of the non-contact sources under investigation in this paper, mounted in a TRANCE device. The top half of the TRANCE device is missing from the photo. Typically the sources are mounted in a nearly enclosed cavity to generate reverberation and thus the directional or omni-directional nature of the radiator is not necessarily important, but rather the cumulative radiated sound energy from each primary source.

\section{Experiment}

As described in the previous section, the acoustic black-hole theory suggests that it is ideal to have the wedge tip approach a zero thickness. It was found that an Electro Discharge Machine (EDM) was able to create thinner wedge tips than a Computer Numerical Control (CNC) machining process. A Mitsubishi FA-10 and $0.254 \mathrm{~mm}$ (0.010 inch) diameter Van-TG brass, plus premium wire was used with 5 skim passes on each $304 \mathrm{~L}$ stainless steel (density $=8030$ $\mathrm{kg} / \mathrm{m}^{3}$, Young's modulus $=193 \mathrm{GPa}$, and Poisson's ratio $=0.24$ ) wedge. The resulting manufactured parts have dimensions of $45 \mathrm{~mm} \times 30 \mathrm{~mm}$ along the bottom surface, including a $30 \times 30 \mathrm{~mm}^{2}$ portion with a constant thickness of $6 \mathrm{~mm}$ and a $15 \times 30 \mathrm{~mm}^{2}$ portion following a 
power-law profile from an original thickness of $6 \mathrm{~mm}$ down to an initial design tip thickness of 75 microns according to Ref. [6]. Using a Nanovea ST400 white light profilometer to measure the surface profile, it was found that the cubic profile tip could be tapered down to a thickness of 156 microns. Attempts to machine the tip thickness to smaller thicknesses failed, resulting in removing material from the wedge taper length and/or significant warping of the shape of the tip. Figure 2 displays the measured side profile, obtained by the profilometer, of the cubic wedge. While not specifically measured, it is assumed by visual inspection that the quadratic and linear wedges were machined down to approximately the same thickness. The thickness of $6 \mathrm{~mm}$ was selected to provide a good initial impedance match for the piezoelectric transducer. The thin plate measured $45 \mathrm{~mm} \times 30 \mathrm{~mm} \times 0.45 \mathrm{~mm}$, with the thickness selected according to the results shown in Fig. 7 of Remillieux et al [6]. The thick plate measured $45 \mathrm{~mm} \times 30 \mathrm{~mm} \times 6.0 \mathrm{~mm}$. The piezoelectric transducers used were made by APC International of type 850 and dimensions of $25.4 \mathrm{~mm}$ diameter by $6.3 \mathrm{~mm}$ thick. Devcon ${ }^{\circledR} 5$ Minute epoxy was used to bond the transducers to the wedges or plate. Figure 3(a) displays a photograph of the transducer assemblies studied.

The transducer wedge/plate under test was mounted to the end of $74 \mathrm{~cm}$ length rod that was connected to a Newport SR50CC rotation stage. Figure 3(b) displays a schematic of the setup. A 12-bit Gage CompuGen 8150 card was used to output a $1 \mathrm{Vpp}$ chirp signal spanning $20 \mathrm{kHz}$ to $100 \mathrm{kHz}$ of $58 \mathrm{~ms}$ duration. A Tabor 9400 power amplifier was used to amplify the chirp signal with a 50 times gain. A GRAS 40BE $6.3 \mathrm{~mm}(0.25$ inch) diameter microphone, with a 4.31 $\mathrm{mV} / \mathrm{Pa}$ sensitivity, was placed $31 \mathrm{~cm}$ from the transducer assembly, perpendicular to the axis of rotation. The axis of rotation is about the axis of the rod as indicated in Fig. 3(b). A PCB 482C 
signal conditioner was used in conjunction with the microphone. A 14-bit Gage CompuScope 14200 card with a $10 \mathrm{MHz}$ sampling frequency was used to record the microphone signals for $65.5 \mathrm{~ms}$. Measurements were made by rotating the transducer assembly at 5 degree increments from 0 to 360 degrees around the axis of the supporting rod, using 244 averages at each position. A signal to noise ratio of at least $40 \mathrm{~dB}$ was obtained over the entire range of frequencies.

The recorded responses were then cross correlated with the chirp signal and the correlation result was time gated to include only the direct arrival of sound (and ignore any reflected energy). A fast Fourier transform is applied to the direct sound signal to obtain a spectrum for the sound radiated from the transducer assembly at each rotation position. Figure 4 displays surface profile plots of the spectra recorded at each rotation angle for the thin plate transducer, the linear wedge, the quadratic wedge, and the cubic wedge transducer. Results for the thick plate are not displayed here as the thin plate easily outperformed the thick plate, as expected from Ref. [6]. An angular averaging was done for each set of spectra to obtain an average sound pressure level spectrum. Additionally, a frequency averaging was done at each angle to determine an average sound pressure level directivity. Figures 5(a) and 5(b) display the average sound pressure level spectra and frequency averaged directivities for each transducer assembly, respectively. It is noted that $0^{\circ}$ corresponds to the tip of the plate/wedge pointing towards the microphone, while $90^{\circ}$ corresponds to the free face of the transducer pointing towards the microphone. Analysis of the results in Figs. 4 and 5 will be presented later on, but the cubic wedge assembly is seen to radiate the most strongly. Thus the scanning laser experiments and analysis will focus on the cubic wedge. 
A PSV400 Polytec scanning laser Doppler vibrometer (SLDV) was used to scan the flat side of the cubic wedge to obtain a modal spectrum. The scanned area spanned $15 \mathrm{~mm}$ by $30 \mathrm{~mm}$, corresponding to the flat side of the wedge taper, with 2,379 scan points for each scan. Figure 5a displays the average vibration spectrum across this scanned area. It is noted that the strong vibration peaks at $22.1 \mathrm{kHz}, 29.0 \mathrm{kHz}, 33.9 \mathrm{kHz}, 38.9 \mathrm{kHz}, 40.5 \mathrm{kHz}, 44.8 \mathrm{kHz}$, and $48.2 \mathrm{kHz}$, corresponding to individual vibration modes, are not at the same frequencies as the peaks in the acoustic radiation spectrum (green curve in Fig. 5a) at $23.3 \mathrm{kHz}, 30.5 \mathrm{kHz}, 36.9 \mathrm{kHz}$, and the wide peak around $50 \mathrm{kHz}$ (strong acoustic spectrum peaks are noted by the black arrows in Fig. 6a). The transducer was then driven with a continuous wave signal at the frequencies corresponding to the strongest vibrational peaks and at the frequencies corresponding to the strongest acoustic radiation peaks. With the use of the SLDV and the Polytec software, the steady-state vibration patterns at these frequencies were obtained. Figures 6(b), 6(c), 6(d), and 6(e) display the vibration patterns, at the time corresponding to the respective maximal amplitudes, for two of the strong vibrational modes $(22.1 \mathrm{kHz}$ and $29.0 \mathrm{kHz})$ and for two of the frequencies at which strong acoustic radiation is measured $(23.3 \mathrm{kHz}$ and $30.5 \mathrm{kHz})$. One can observe that the two patterns corresponding to strong vibrational modes each possess multiple antinodes along the length of the wedge tip. In contrast, the two patterns at frequencies with strong acoustic radiation have a single antinode along the wedge tip.

The scanning laser was also used to image the vibration of the flat side of the cubic wedge as a $75 \mathrm{kHz}$ pulse was emitted by the transducer. The vibrational amplitude at the tip of the wedge 
was found to be 20 times larger than the vibrational amplitude on the face of the wedge directly opposite to the transducer (the $6 \mathrm{~mm}$ thick portion). Animations of the vibrations can be seen in Supplementary Videos 1 and 2. The difference in these animations is that the color scale has been clipped in the second one in order to see the vibration in the thick portion of the wedge.

A single laser of a PSV-500-3D Polytec scanning laser Doppler vibrometer was used to scan the radiated acoustic wave field generated by the cubic wedge transducer. A chirp signal, spanning $50 \mathrm{kHz}$ to $100 \mathrm{kHz}$ was used as the excitation signal and the laser detected the pressure field by virtue of the acousto-optic effect. See References [10] and [11] for examples of laser Doppler vibrometers being used to image sound fields. This method extracts an instantaneous magnitude and phase, averaged over the entire path of the laser's emitted and reflected light, and thus can only obtain a $2 \mathrm{D}$ projection of the $3 \mathrm{D}$ sound field it scans. The RMS magnitude of the velocity over the entire chirp bandwidth for each scan point is displayed in Fig. 8. The reason for the tilt of the cubic wedge in Fig. 8 is due to limitations in the setup for this measurement. The scan points that caused the laser to shine on portions of the wedge and/or the transducer were omitted from the display. For the acousto-optic effect to work well, the laser must reflect off of a rigid surface that has been coated with reflective tape. In this case we used a thick aluminum block as the rigid reflector.

\section{Discussion}

In general, the peak radiation from each of the transducer assembly results displayed in Figs. 4 and 5 is that radiated at $90^{\circ}$. This radiation is either directly radiated from the free face of the 
transducer, from the wedge tip (that faces the same direction as the free face of the transducer), or a combination of the two. Additionally, significant radiation is seen at $77 \mathrm{kHz}$, the resonance of the radial mode of the transducer. The overall sound pressure levels of the various assemblies are $102.3 \mathrm{~dB}, 104.2 \mathrm{~dB}, 105.6 \mathrm{~dB}$, and $107.3 \mathrm{~dB}$ for the thin plate, linear wedge, quadratic wedge, and cubic wedge assemblies, respectively. However, these overall sound pressure levels are principally governed by the respective peaks in the spectra, and do not reflect the large increases in the low and high frequency radiation portions of the spectra. Figure 8 displays the difference between the spectra radiated by each wedge assembly compared to the spectrum radiated by the thin plate $(>0 \mathrm{~dB}$ represents increases in radiation over the thin plate). To compute the average radiation improvements, the difference spectra in Fig. 8 must be converted to pressures, and then the average pressure difference may be converted back into a decibel value. The average decibel improvement in radiation of the wedge assemblies over the thin plate assembly are $1.7 \mathrm{~dB}, 3.6 \mathrm{~dB}$, and $8.4 \mathrm{~dB}$ for the linear, quadratic, and cubic wedge assemblies, respectively.

As noted in Section 2, the vibration patterns measured at the frequencies corresponding to the peak acoustic radiation each have strong antinodes at the tip of the cubic wedge, whereas the vibrational patterns corresponding to the strongest structural spectrum peaks have several antinodes along the tip of the cubic wedge. A Rayleigh integral analysis of each of the 4 vibrational patterns displayed in Figs. 5(b)-5(e) can be done, assuming the wedge has an infinite baffle surrounding it, in order to determine which of these patterns should couple to acoustic radiation strongly. This analysis is admittedly crude but it provides insight into the efficiency of 
the modal radiation. If we consider a field point directly above the wedge tip $\left(90^{\circ}\right)$, we can integrate over the scanned area using the infinite baffle Rayleigh integral [12],

$$
\hat{p}(R)=\frac{j \omega \rho_{0}}{2 \pi} \iint_{S} \hat{u}_{n}(x, y) \frac{e^{-j k R}}{R} d S
$$

where $\hat{p}$ is the acoustic pressure, $R$ is the distance from the field point to $(x, y), j$ is the imaginary number $\sqrt{-1}, \omega$ is the angular frequency, $\rho_{0}$ is the density of air, $S$ is the spatial surface on the wedge over which to integrate, $\hat{u}_{n}$ is the complex amplitude of the normal surface velocity, $(x, y)$ are the individual coordinates of each point on the spatial surface of the wedge, and $k$ is the acoustic wavenumber. The Rayleigh integral sound pressure level amplitudes (re 20 $\mu \mathrm{Pa}$ ), at a distance of $R=31 \mathrm{~cm}$, are $64.2 \mathrm{~dB}, 77.6 \mathrm{~dB}, 64.9 \mathrm{~dB}$, and $80.7 \mathrm{~dB}$ for the vibrational patterns at $22.1 \mathrm{kHz}, 23.3 \mathrm{kHz}, 29.0 \mathrm{kHz}$, and $30.5 \mathrm{kHz}$, respectively. Thus, at $90^{\circ}$, one would expect a strong acoustic radiation improvement of approximately $13.4 \mathrm{~dB}$ at $23.3 \mathrm{kHz}$ over that radiated by the mode at $22.1 \mathrm{kHz}$ and an improvement of approximately $15.8 \mathrm{~dB}$ at $30.5 \mathrm{kHz}$ over that radiated by the mode at $29.0 \mathrm{kHz}$, despite the relatively low vibrational amplitudes of the strong acoustic modes $(23.3 \mathrm{kHz}$ and $30.5 \mathrm{kHz})$ versus the strong vibrational modes $(22.1$ $\mathrm{kHz}$ and $29.0 \mathrm{kHz}$ ). Thus the strong acoustic radiation from the cubic wedge, at frequencies below $50 \mathrm{kHz}$, is due in large part to the efficient radiation of certain structural modes of the wedge.

The acoustic black-hole theory for vibration mitigation suggests that this effect works best at high frequencies [3], though there is an upper frequency limit that the structural wavelength be larger than the wedge length. In the black-hole theory, the goal is to reduce the propagation 
speed of the structural waves to zero so that they are damped at the thin wedge tip before reflecting back to the bulk structure. However, one might assume that some of the vibrational energy loss in the black-hole is radiated away as sound energy. Here we have found that improvements in sound radiation were found essentially at all frequencies studied ( $20 \mathrm{kHz}$ to 100 $\mathrm{kHz}$ ). The critical frequency for flexural waves in a plate occurs when the flexural wave speed equals the fluid sound speed (see equation (4.41) in Ref. [13]). Below this frequency, any significant radiation from the plate will be from efficient coupling of certain modes of the plate (i.e. edge modes and corner modes) to sound radiation. Plates generally radiate most efficiently at this frequency and above. With the wedge of cubic profile, the thickness at the tip is $156 \mu \mathrm{m}$ and a plate of this thickness would have a critical frequency of $79.6 \mathrm{kHz}$. The critical frequency is inversely proportional to the thickness of the plate. Thus waves of frequencies below $80 \mathrm{kHz}$ will be supercritical before reaching the wedge tip and may radiate before reaching the tip. The flexural wave impedance, for a plate of $156 \mu \mathrm{m}$ thickness, equals the characteristic impedance of air (415 Rayl.) at $52.9 \mathrm{kHz}$. Thus the gains in acoustic radiation at high frequencies are likely due to the combination of a good impedance matching of flexural waves with the impedance of air and because the waves are traveling at or near supercritical speeds. The flexural wave speed decreases with decreasing wedge thickness and thus it is possible that a finite thickness wedge tip is desired for optimal sound radiation (so that the flexural wave speed remains supercritical). As discussed earlier, there are many peak frequencies at which the wedge with a cubic profile radiates well that correspond to modes of the wedge. The dominance of modal radiation would be expected below the critical frequency. 
A comparison of the radiation from the wedge of cubic profile to that of a bare piezoelectric transducer, as done in Ref. [6], could not be easily accomplished since it is challenging to rotate a freely vibrating transducer without mounting it to something. However, a measurement of a bare, cylindrical piezoelectric transducer, the same as those used above, with the flat face facing the microphone, was conducted. This measurement was compared to the measurement from the wedge of cubic profile at the corresponding angular rotation position. The wedge of cubic profile radiated more energy at essentially all frequencies, ranging from $20 \mathrm{~dB}$ gains at peak frequencies such as $23 \mathrm{kHz}, 30 \mathrm{kHz}$, and $98 \mathrm{kHz}$ to gains of $5 \mathrm{~dB}$ at most of the frequencies in between. The difference in the overall sound pressure level in the two measurements was $5.2 \mathrm{~dB}$, largely representing the difference between the two transducer configurations at their resonance frequencies. The spectral shape of the bare piezoelectric transducer closely followed that for configuration 1 depicted in Fig. 5 of Ref. [6], with a single strong peak at the transducer's resonance near $75 \mathrm{kHz}$, while the wedge of cubic profile was found to have several peak frequencies, qualitatively similar to configuration 4 in Ref. [6].

It is noted that the radiation pattern depicted in Fig. 7 looks like that of a quadrupole. This acousto-optic image only images the near field radiation, and thus the far field radiation pattern may not necessarily look the same. However, it appears that three of the radiated beams of sound visible in Fig. 7 correspond to the peaks in Fig. 5(b) at $45^{\circ} 245^{\circ} 317^{\circ}$, which beams are generated by wedge tip vibration. Radiation at $0^{\circ}$ and $180^{\circ}$ appears to be minimal in Fig. 7, as shown in Fig. 5(b), because there is no significant vibration of the transducer or the wedge in those directions. Radiation in the $90^{\circ}$ and $270^{\circ}$ directions is not as apparent in Fig. 7, though 
there is some indication of radiation in the $90^{\circ}$ direction off of the free face of the piezoelectric transducer, which is interfering with the radiation one might expect in the $120^{\circ}$ direction.

\section{Conclusions}

It has been shown experimentally here that a wedge of power law profile, specifically that of cubic profile, can provide an efficient means of coupling vibrational energy generated by a piezoelectric transducer to ultrasonic radiation. Some of this increased radiation at lower frequencies is due to the efficient radiation of certain vibrational modes, with the highest vibrational amplitudes at the thin tip of the cubic wedge. The increased radiation at higher frequencies is likely due to efficient radiation of supercritical flexural waves and because the impedance contrast with the surrounding fluid is less pronounced. As the waves travel down the cubic wedge profile, the waves increase in amplitude by a factor 20 due to the decrease in impedance of the wedge, the wave speed slows down due to the decreasing thickness of the wedge trapping the waves near the wedge tip, and they subsequently strongly radiate energy at the wedge tip.

\section{Acknowledgements}

We gratefully acknowledge the support of the U.S. Department of Energy through the LANL/LDRD Program and the Foundation for Polish Science (FNP) within the scope of the WELCOME Programme - project no. 2010-3/2.

\section{References}


[1] M. C. Remillieux, B. E. Anderson, P.-Y. Le Bas, T. J. Ulrich, M. R. Haberman, and J. Zhu "Review of air-coupled transduction for nondestructive testing and evaluation," Acoust. Today 10(3), 36-45 (2014).

[2] V. V. Krylov and R. E. T. B. Winward, "Experimental investigation of the acoustic black hole effect for flexural waves in tapered plates," J. Sound Vib. 300(1-2), 43-49 (2007).

[3] V. V. Krylov, "Acoustic black holes: Recent developments in the theory and applications,” IEEE Trans. Ultrason. Ferroelectr. Freq. Control 61(8), 1296-1306 (2014).

[4] M. A. Mironov, "Propagation of a flexural wave in a plate whose thickness decreases smoothly to zero in a finite interval," Sov. Phys. Acoust. 34(3), 318-319 (1988).

[5] V. V. Krylov, "Localized acoustic modes of a quadratic solid wedge," Mosc. Univ. Phys. Bull. 45(6), 65-69 (1990).

[6] M. C. Remillieux, B. E. Anderson, P.-Y. Le Bas, and T. J. Ulrich, "Improving the air coupling of bulk piezoelectric transducers with wedges of power-law profiles: A numerical study,” Ultrasonics 54(5), 1409-1416 (2014).

[7] P.-Y. Le Bas, T. J. Ulrich, B. E. Anderson, and J. J. Esplin, “A high amplitude, time reversal acoustic non-contact excitation (TRANCE)," J. Acoust. Soc. Am. 134(1), EL52EL56 (2013).

[8] M. Fink, “Time reversed acoustics,” Phys. Today 50, 34-40 (1997).

[9] B. E. Anderson, M. Griffa, C. Larmat, T. J. Ulrich, and P. A. Johnson, "Time reversal," Acoust. Today 4(1), 5-16 (2008). 
[10] Y. Wang, J. Tyrer, P. Zhihong, and W. Shiquan, "Measurement of focused ultrasonic fields using a scanning laser vibrometer," J. Acoust. Soc. Am. 121(5), 2621-2627 (2007).

[11] A. Torras-Rosell and S. Barrera-Figueroa, "Sound field reconstruction using acoustooptic tomography,” J. Acoust. Soc. Am. 131(5), 3786-3793 (2012).

[12] N. Etaix, M. Fink, and R. K. Ing, “Acoustic imaging device with one transducer," J. Acoust. Soc. Am. 131(5), EL395-EL399 (2012).

[13] F. Fahy and P. Gardonio, Sound and Structural Vibration, Radiation, Transmission and Response, $2^{\text {nd }}$ ed. (Academic, Oxford, U.K., 2007), Chapter 5.

\section{List of Captions}

Fig. 1. Photograph of the bottom half of a Time Reversal Acoustic Non-Contact Excitation (TRANCE) device with 32 cubic wedge transducers mounted inside. The $2.54 \mathrm{~cm} \times 2.54 \mathrm{~cm}$ screw hole pattern in the optical table in the background provides a scale reference.

Fig. 2. Cubic wedge side profiles obtained from a white light profilometer. (a) Zoomed out with $\mathrm{x}$ and $\mathrm{y}$ axes to scale. (b) Zoomed in image, with the $\mathrm{x}$ scale enlarged.

Fig. 3. (a) Photograph of the piezoelectric transducers and wedges/plates used in this study. (b) Schematic of the setup for measuring directivity.

Fig. 4. Sound pressure level (SPL) versus frequency and angle for the thin plate, linear wedge, quadratic wedge, and cubic wedge assemblies in (a), (b), (c), and (d), respectively. 
Fig. 5. (a) Frequency response (angular averaged SPL) for the four assemblies indicated. (b) Directivity (frequency averaged SPL) for the four assemblies indicated.

Fig. 6. (a) Average structural vibration frequency response for the cubic wedge. (b)-(e) Sample vibration modes at the frequencies indicated in the subplot titles measured on the opposite (flat) side of the taper portion of the cubic wedge.

Fig. 7. Acousto-optic image of the RMS magnitude of the $50 \mathrm{kHz}$ to $100 \mathrm{kHz}$ bandwidth wave field radiated by the cubic wedge transducer. Black colored lines were added to outline the piezoelectric transducer and the wedge.

Fig. 8. Frequency response difference spectra for the linear, quadratic, and cubic wedge assemblies compared to the thin plate assembly as a reference. $>0 \mathrm{~dB}$ indicates higher radiation levels compared to the thin plate. 

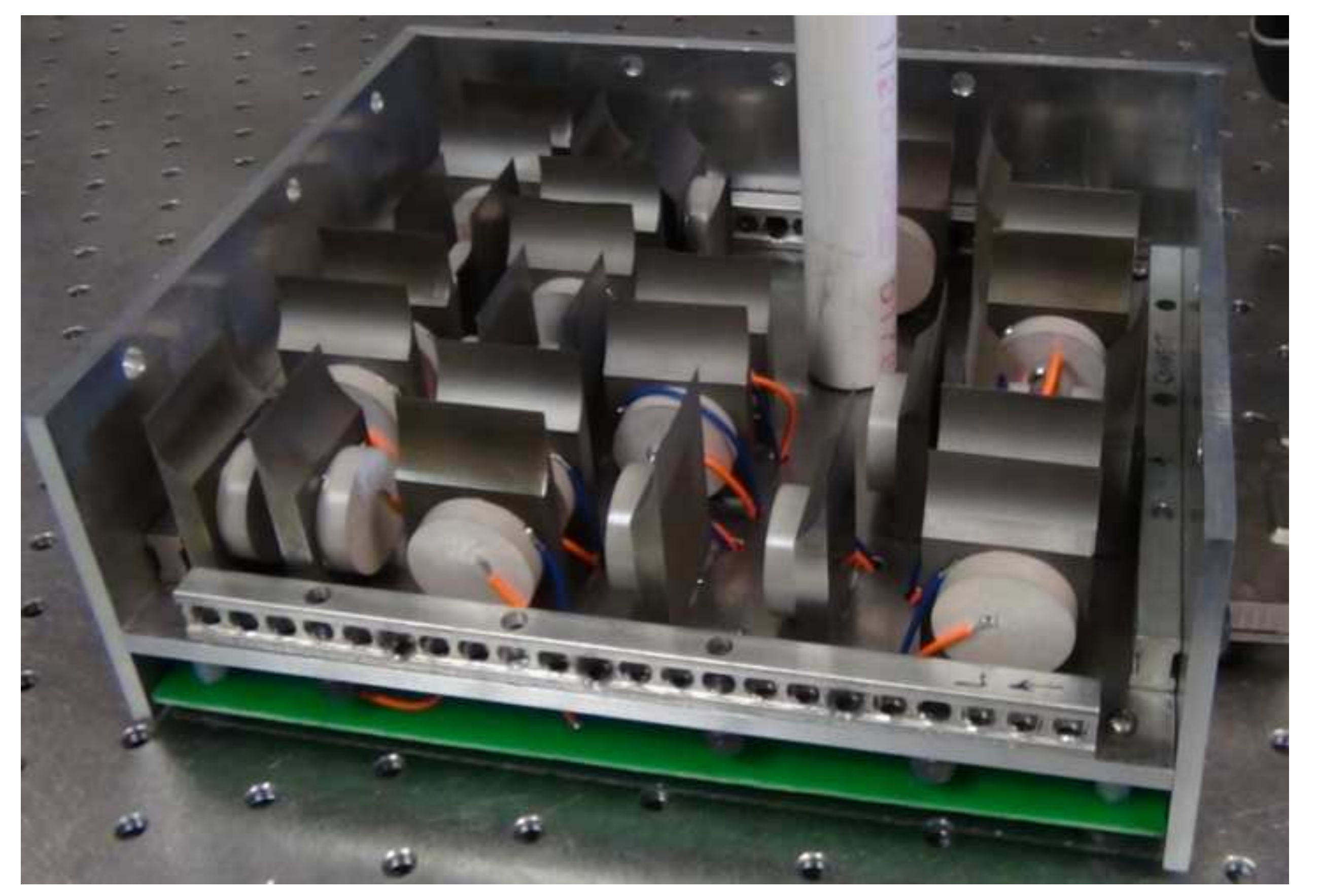
(a)

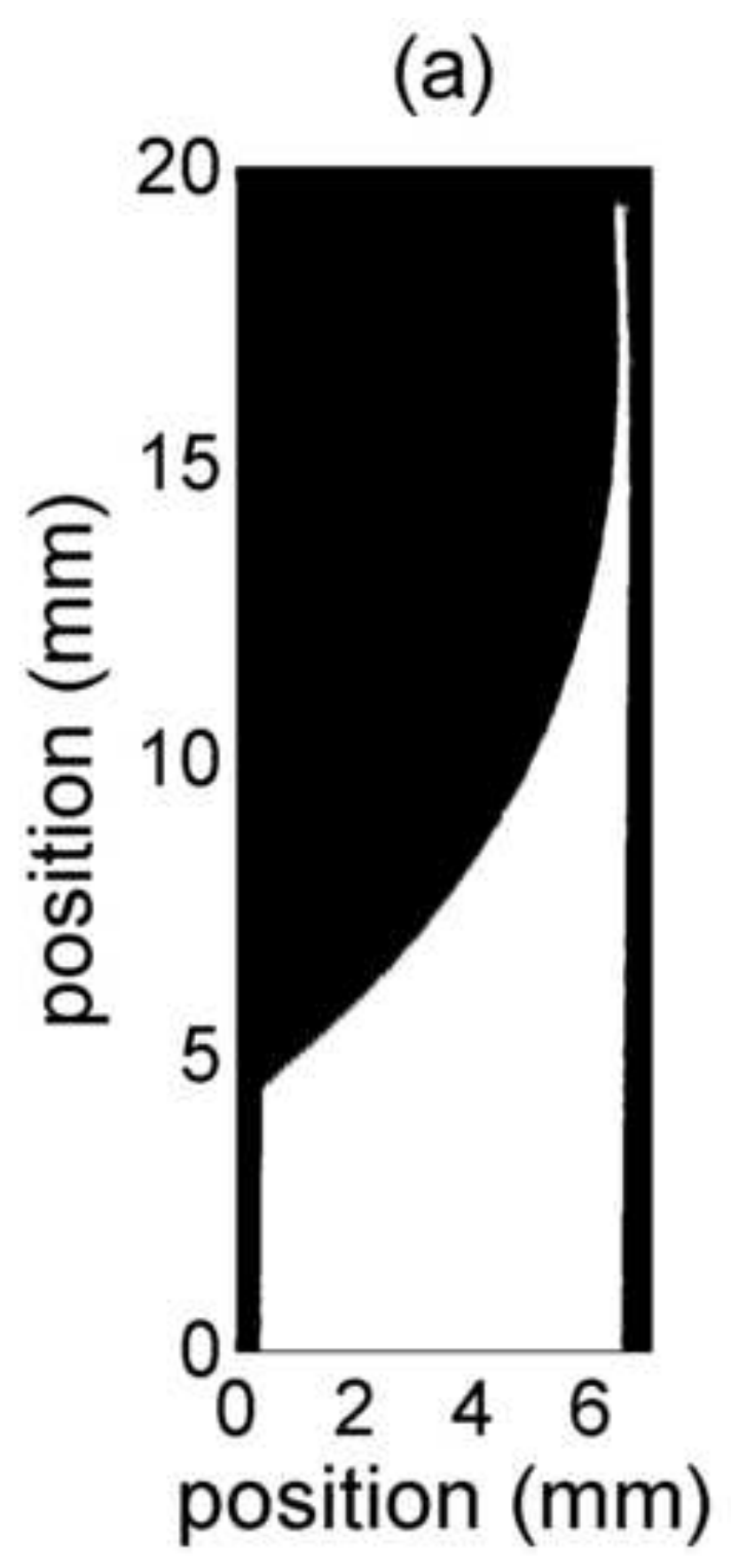

(b)

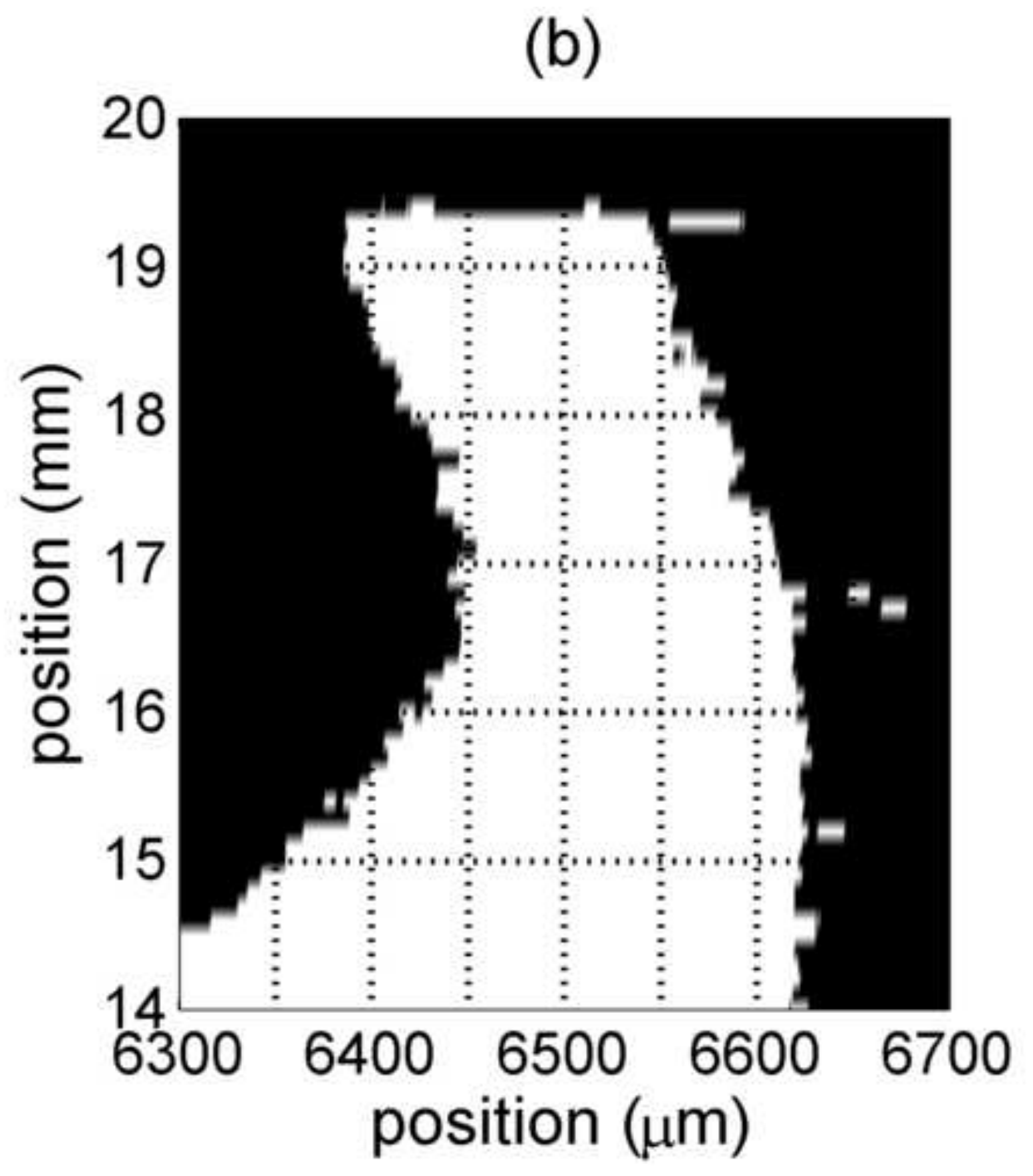



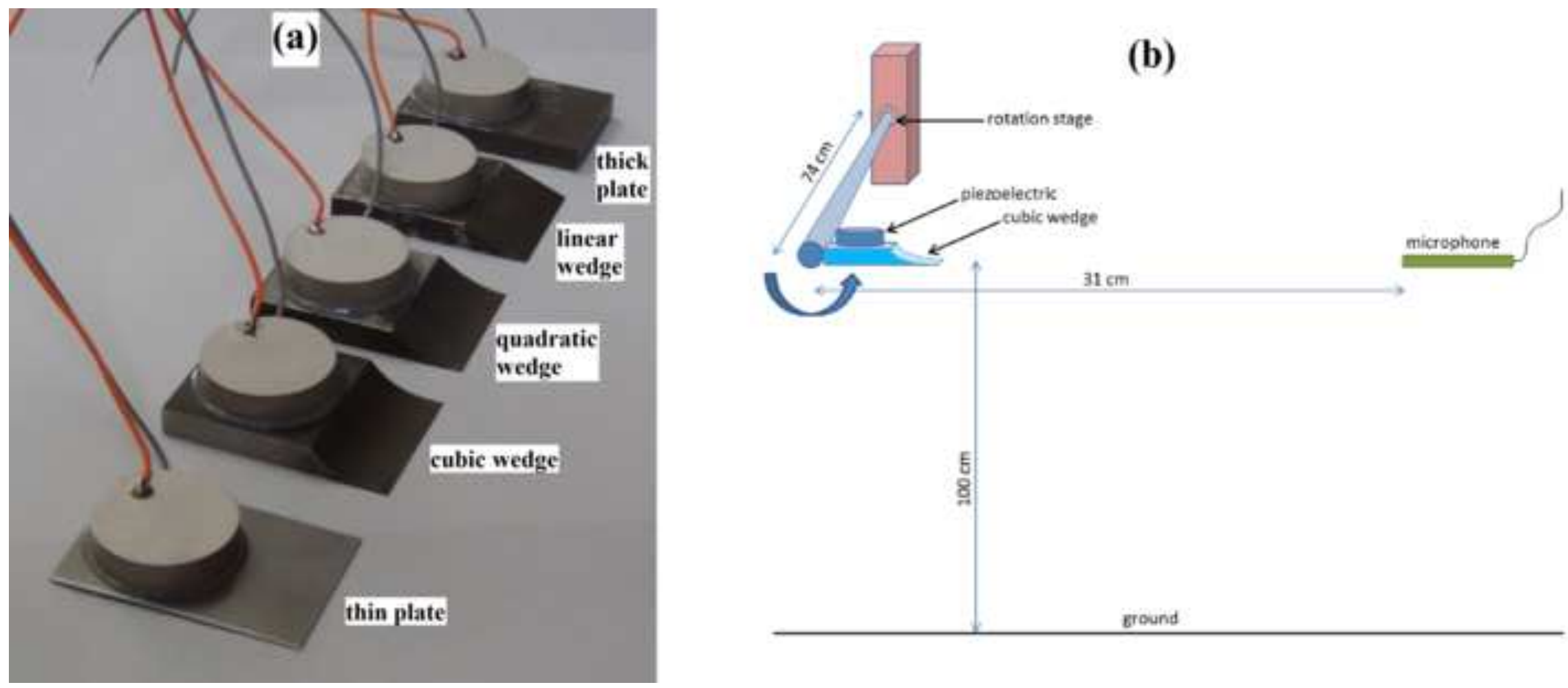
(a) Thin Plate

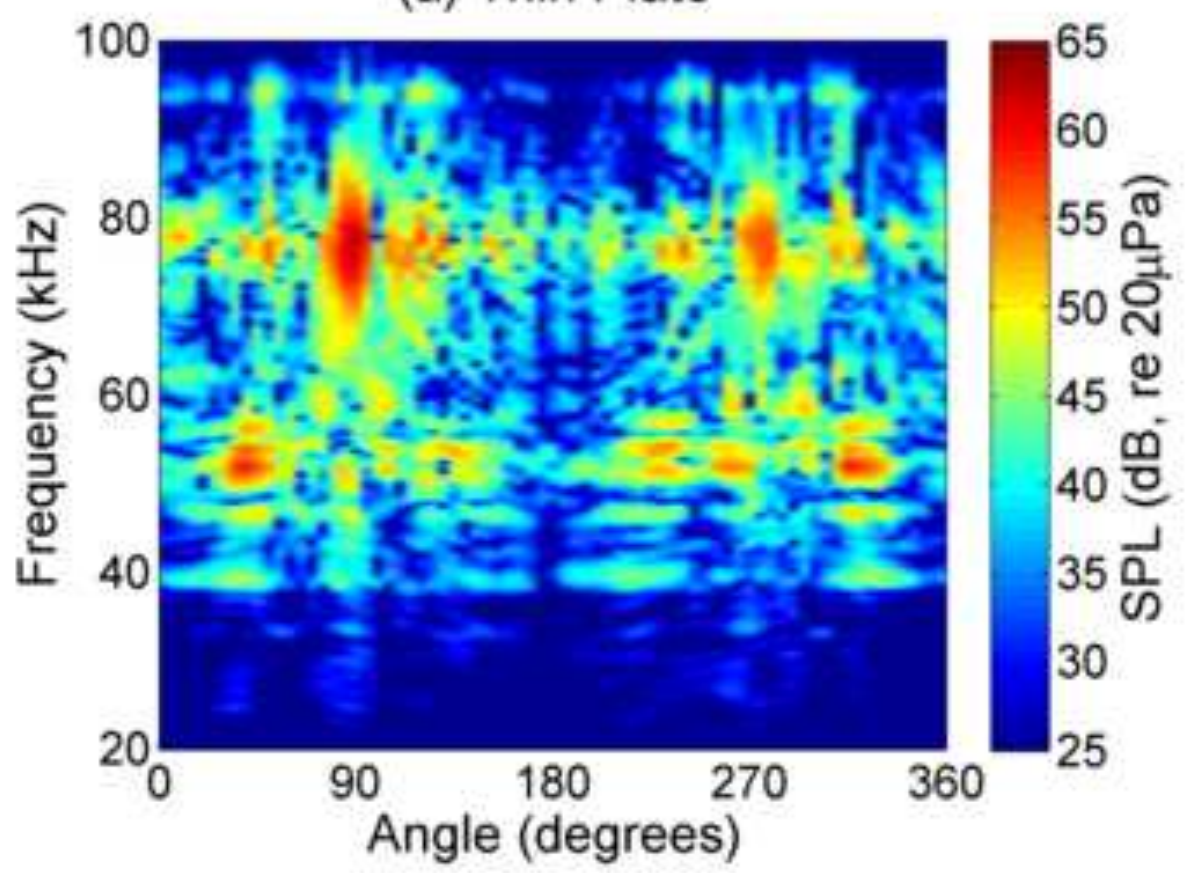

(c) Quadratic Wedge

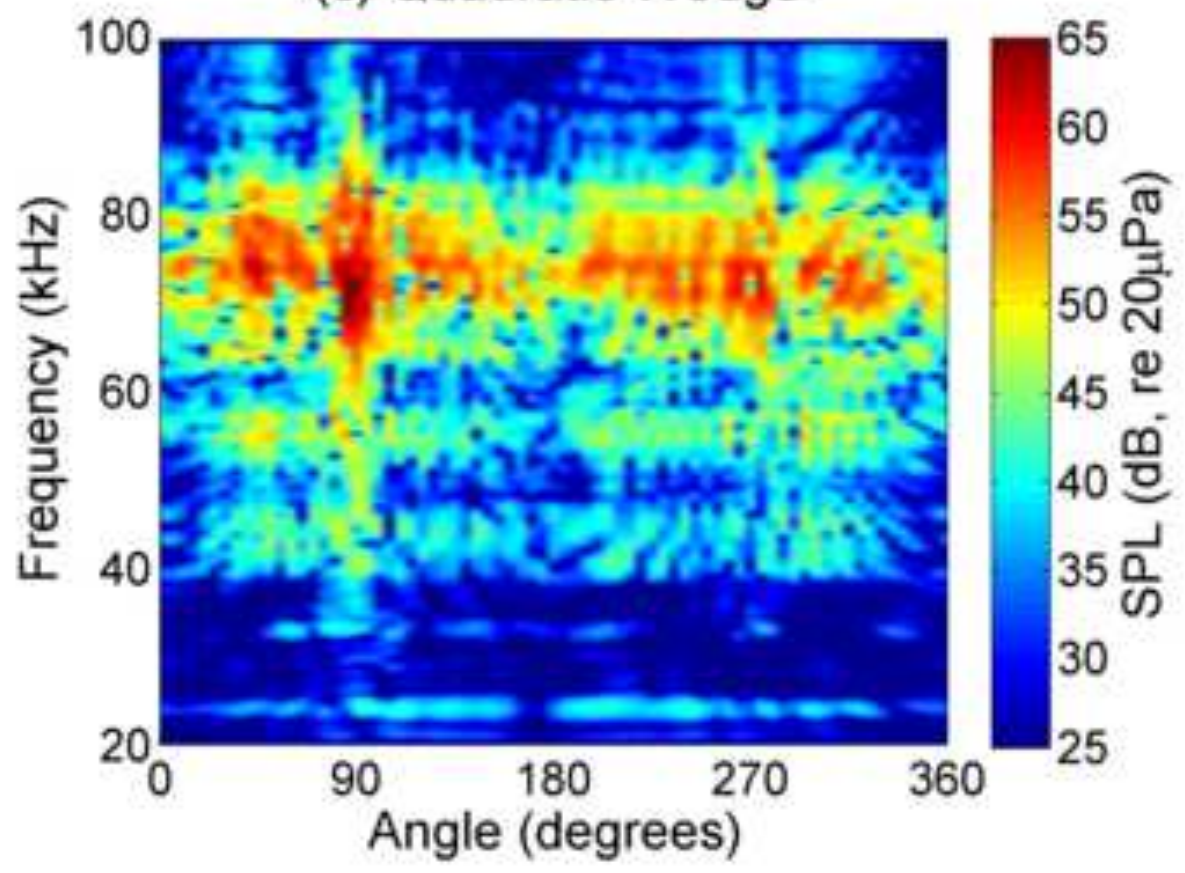

(b) Linear Wedge

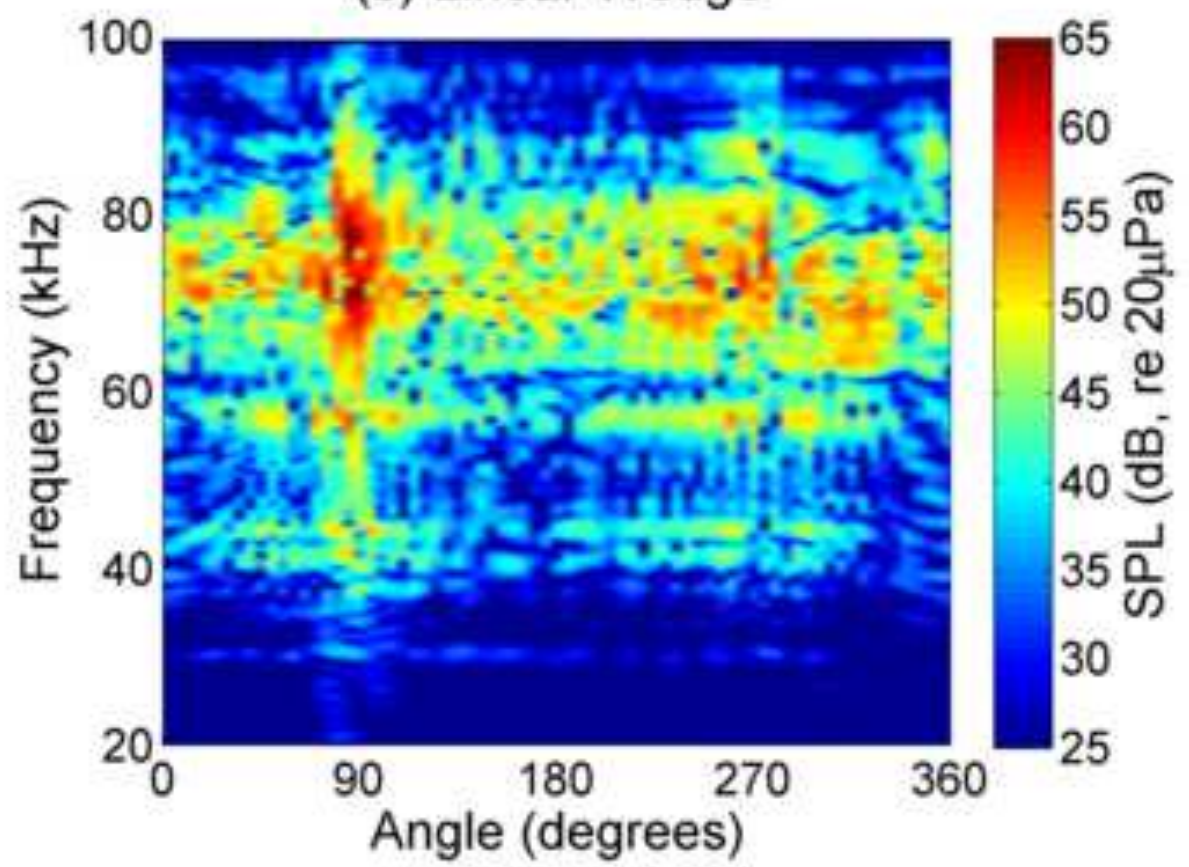

(d) Cubic Wedge

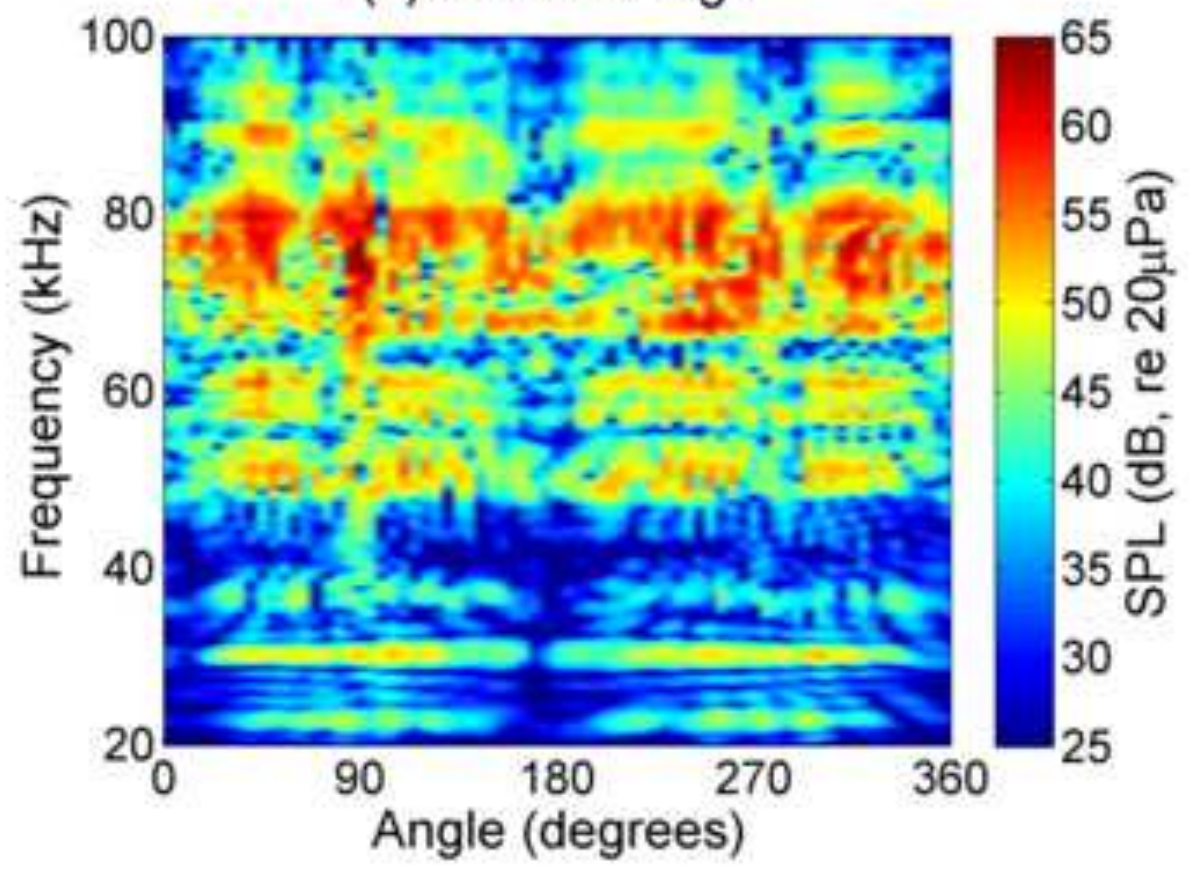


(a)

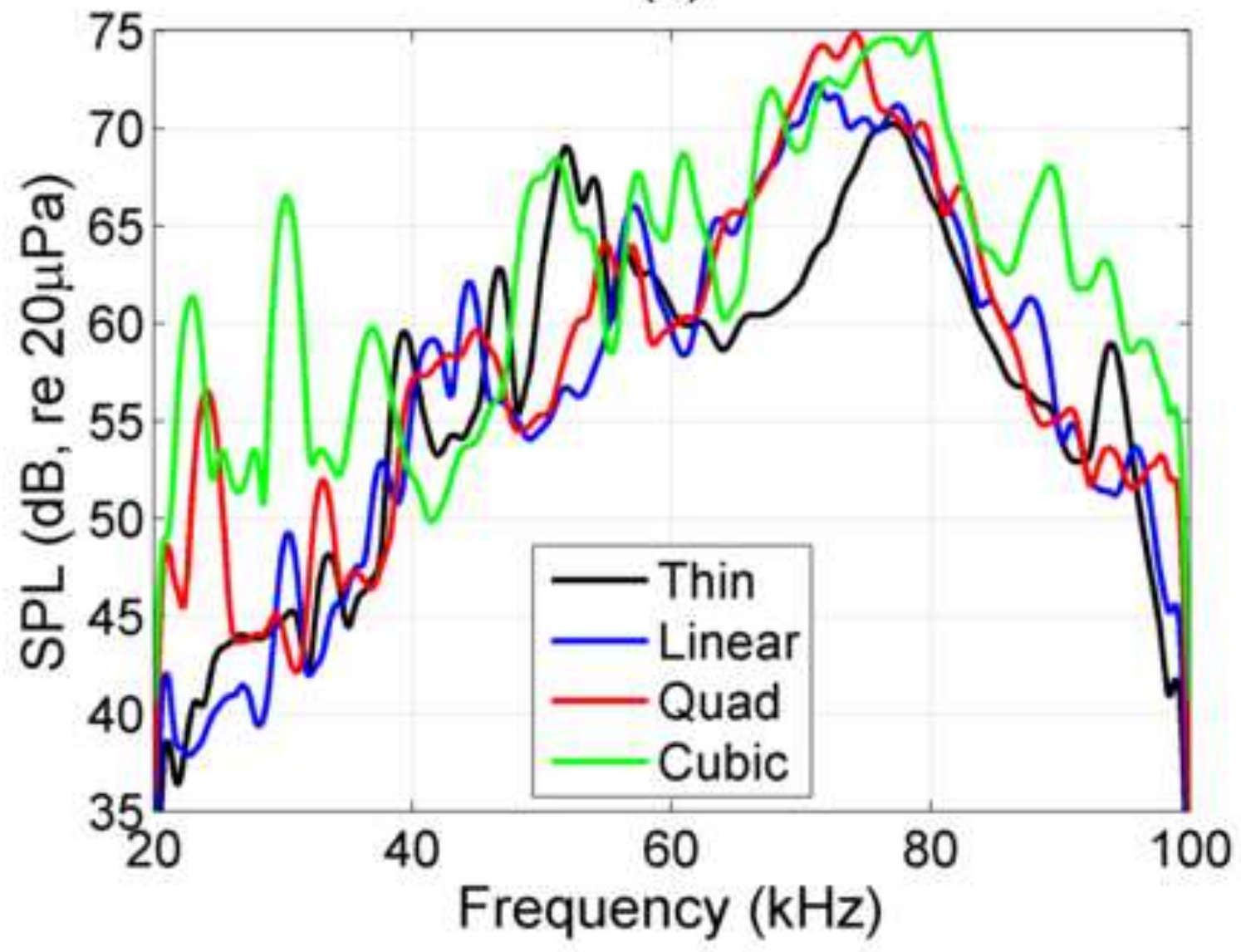

(b)

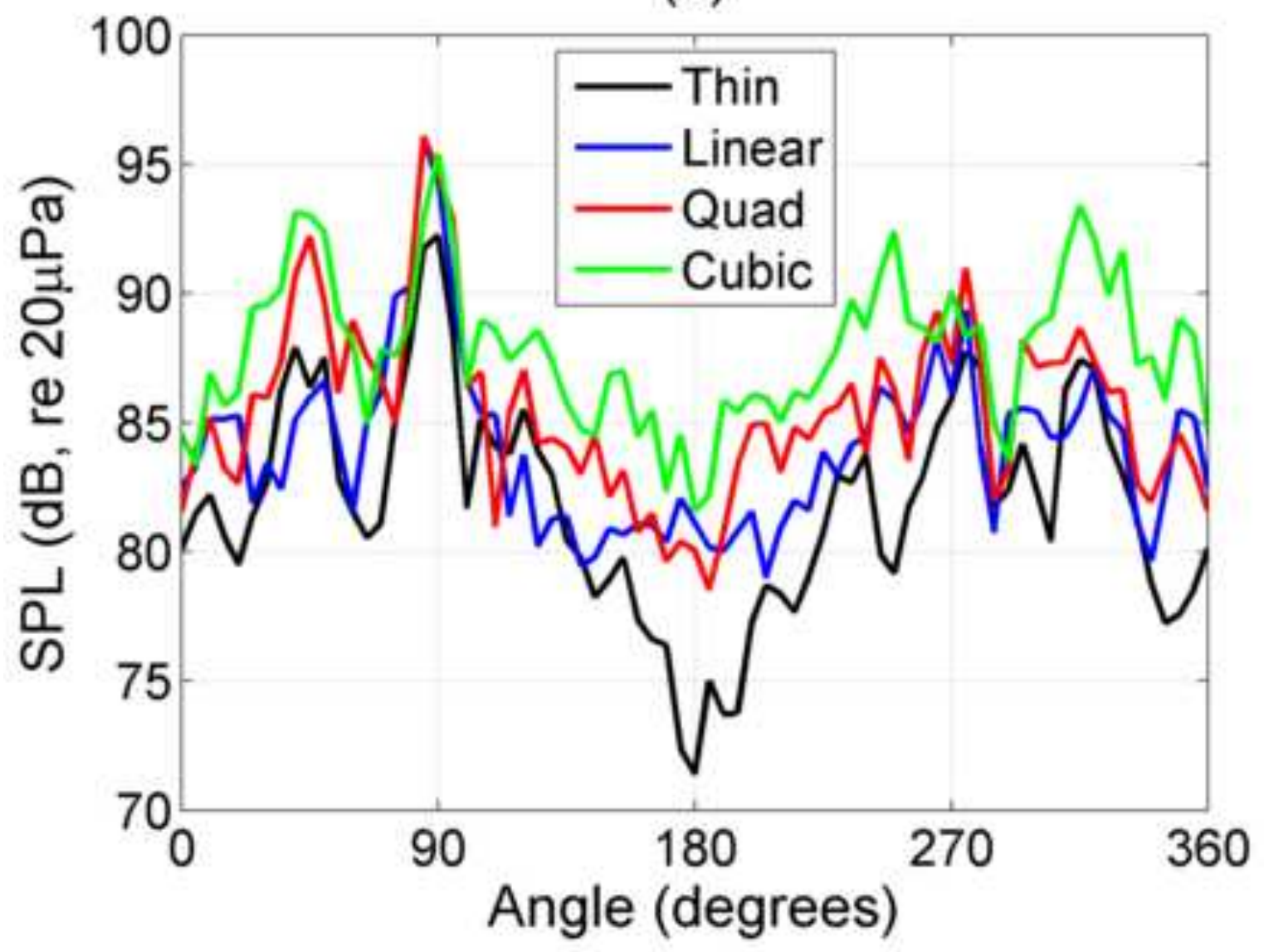


(a)

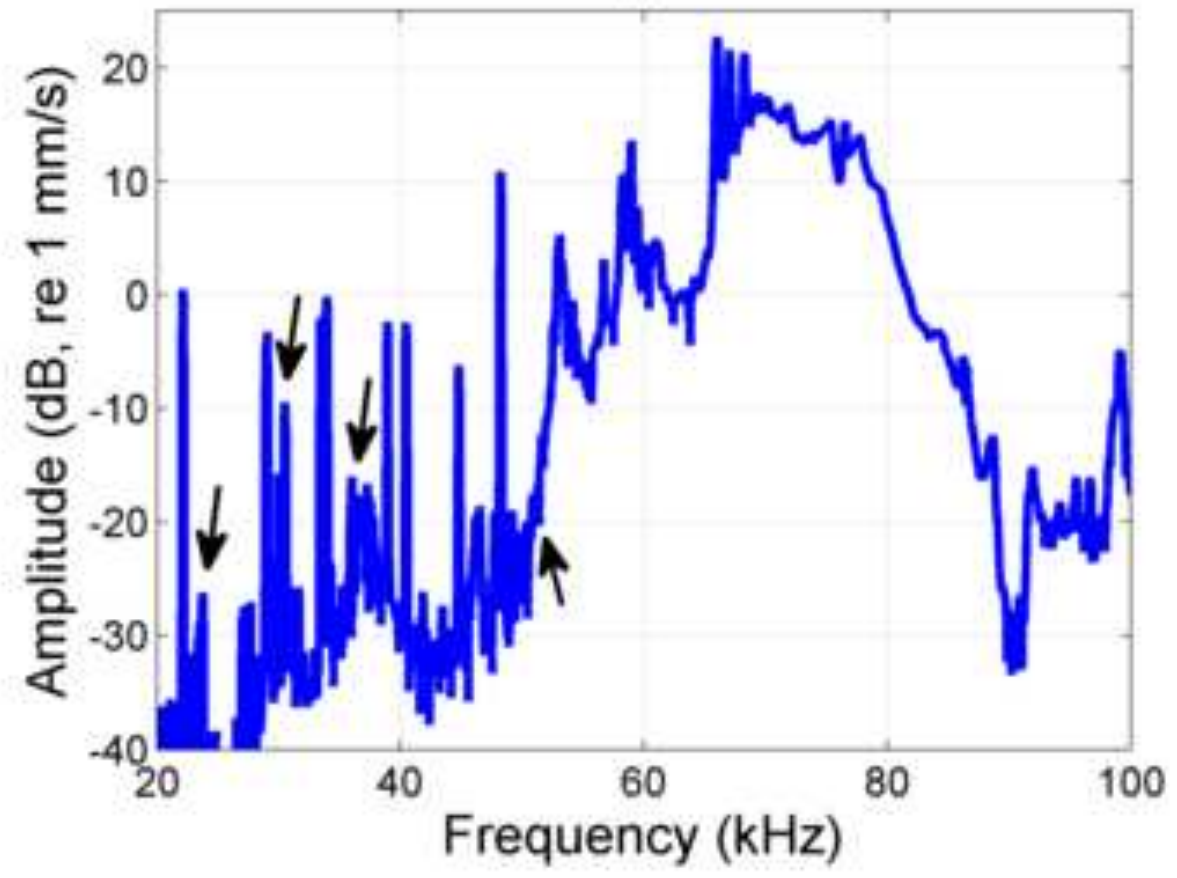

(b) $22.1 \mathrm{kHz}$

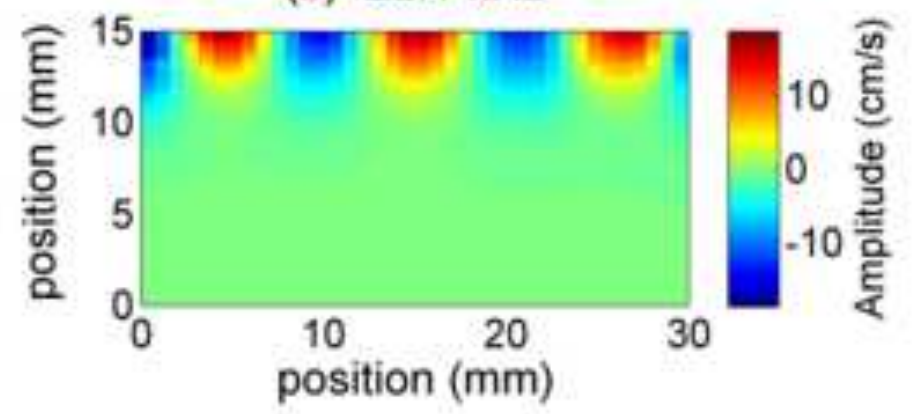

(c) $23.3 \mathrm{kHz}$
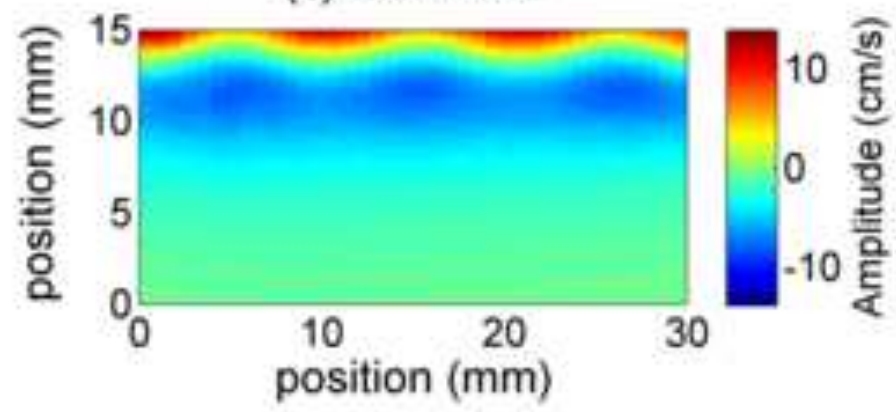

(d) $29.0 \mathrm{kHz}$

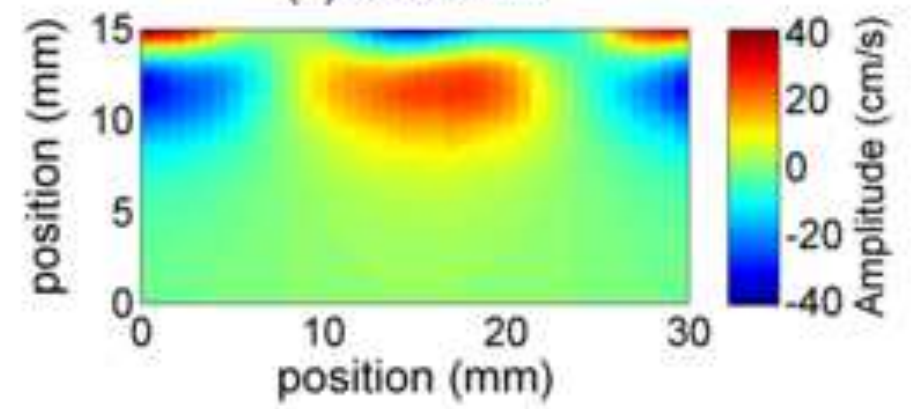

(e) $30.5 \mathrm{kHz}$

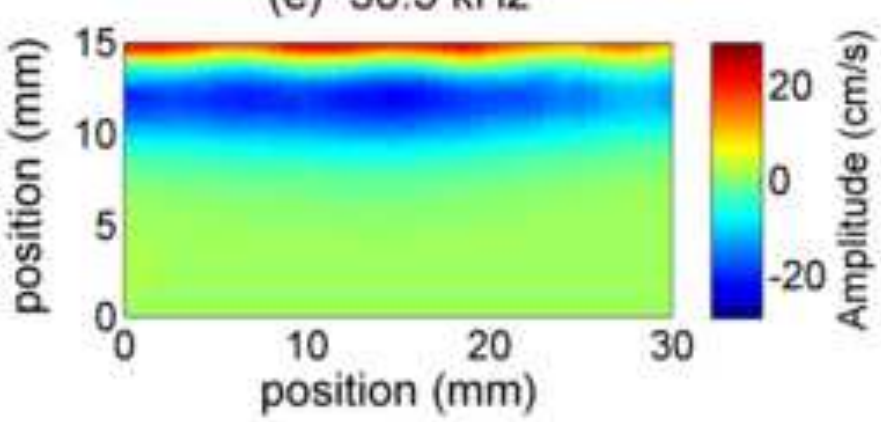




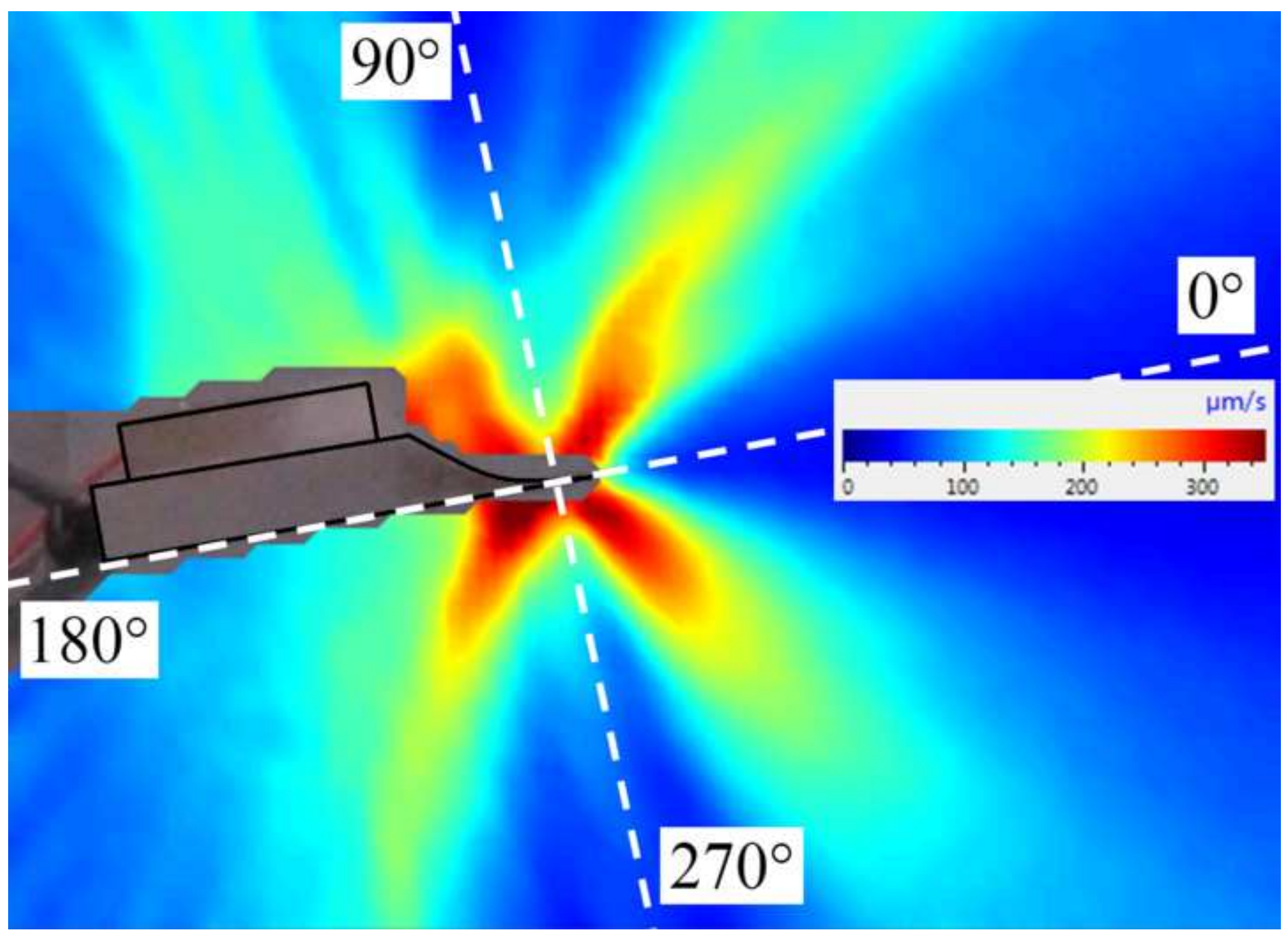




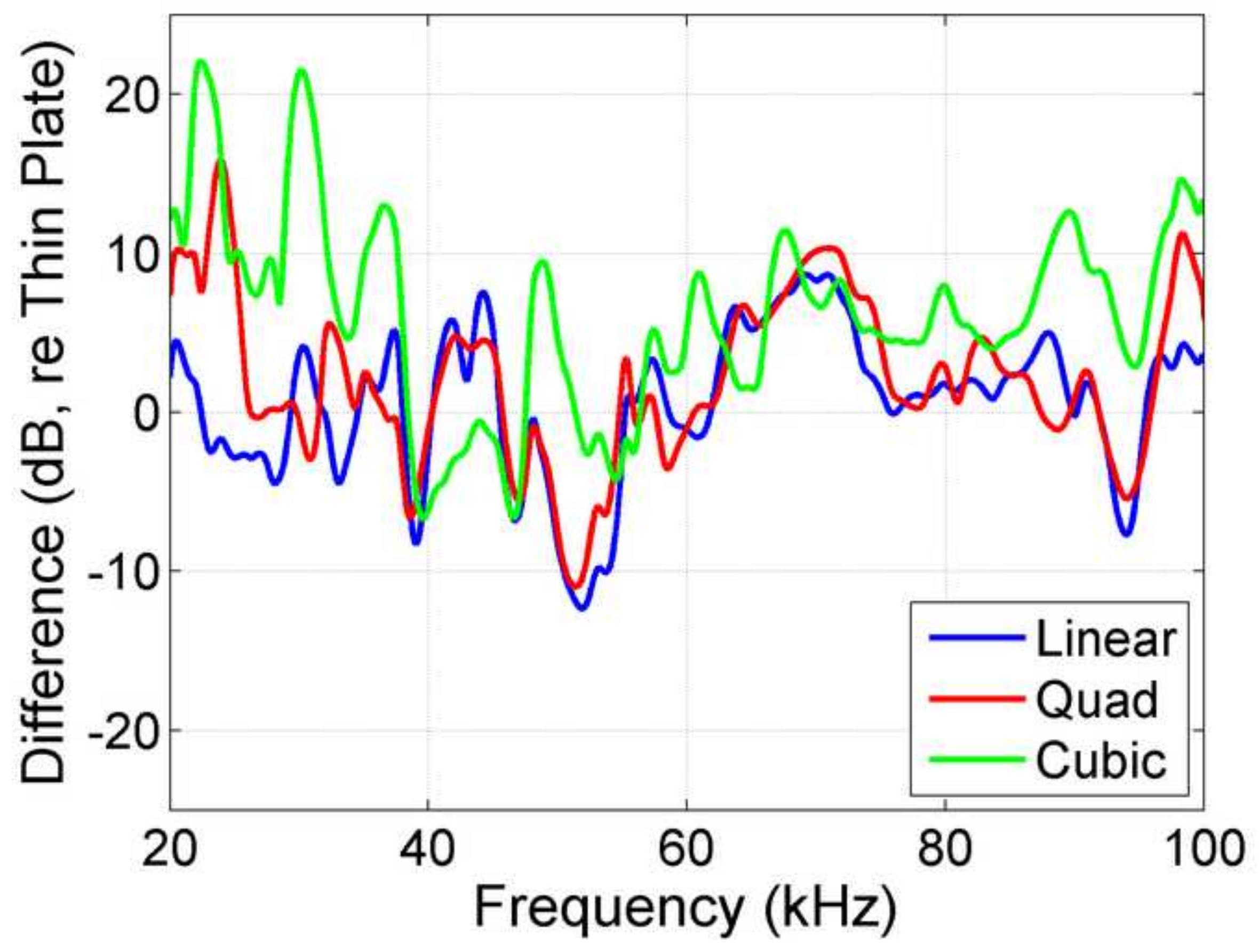

